\title{
Coordinating Multi-layer MPC for Complex Water Systems
}

\author{
Congcong Sun ${ }^{1}$, Vicenç Puig ${ }^{1}$, Gabriela Cembrano ${ }^{1,2}$ \\ 1. Advanced Control Systems Group at the Institut de Robòtica i Informàtica Industrial, Llorens i Artigas, 4-6, Barcelona, Spain. \\ E-mail: vicenc.puig@upc.edu \\ 2. CETaqua, Water Technology Centre at Esplugues 75, Cornellà de Llobregat, Barcelona, Spain. \\ E-mail: cembrano@iri.upc.edu
}

\begin{abstract}
In this paper, coordinating technique for the multi-layer model predictive control (MPC) of complex water network is proposed. A multi-layer topology structure resulting from a functional decomposition of water network is briefly presented. Inside each layer, a MPC based controller is used. Between the related two layers, a multi-layer coordinating mechanism is provided to generate control strategies which consider objectives and time scales of both layers. Optimal Path Method (OPM) is used to estimate aggregated water price for the whole layer. The case study is the Catalunya Regional Water Network. Simulation results based on an aggregate model of this network are provided.
\end{abstract}

Key Words: Multi-layer MPC, Coordinating Mechanism, OPM

\section{INTRODUCTION}

From a functional perspective, a complex water network can be basically organized into two layers [1]:

- Supply layer, composed of water sources, large reservoirs and also natural aquifers.

- Transportation layer, linking water treatment with reservoirs distributed all over the city.

Both layers should be operated at different time scale because of the different dynamics according to the specified objectives. In general, these layers are often separately operated $[2,4,1]$. Coordinating the different layers for ecological effect improvement and also sustainable usages of water resource is one of the main motivations for the research reported in this paper.

MPC has been proven to be one of the most effective control strategies for the global optimal operational control of large-scale water networks $[1,2,3]$.

The paper continues the research presented in [10], being the main contribution a multi-layer MPC coordinating scheme for complex water networks with more accurate aggregated water price estimated by OPM. The proposed strategy will coordinate the MPC controllers in the supply and transportation layers by means of a hierarchical sequence of optimizations and constraints going from the upper to the lower layer.

Organization of this paper is: Section 2 introduces the control oriented modelling methodology proposed for complex water networks. In Section 3, MPC, multi-layer MPC and OPM are outlined. In Section 4 , the formulation of MPC

This research has been partially funded by CDTI (MCyT) project HIDROPTIM IDI-20100722, the DGR of Generalitat de Catalunya (SAC group Ref. 2009/SGR/1491), the AGAUR by an FI grant and by EFFINET grant FP7-ICT-2012-318556 of the European Commission. and temporal multi-layer coordination technique is presented. In Section 5, the case study based on the Catalunya Regional Water Network is described. In Section 6, the simulation results of the proposed approach to Catalunya Regional Water Network are outlined. Finally, in Section 7 , the main conclusions are presented

\section{CONTROL ORIENTED MODELLING METHODOLOGY}

Modelling of the different elements appear in a complex water system is provided in this section.

\subsection{Dams, Tanks and Reservoirs}

The balance expression relating the stored volume $v$, the manipulated inflows $q_{\text {in }}^{i, j}$ and outflows $q_{\text {out }}^{i, l}$ (including the demand flows as outflows) for the $i$-th storage element can be written as:

$$
v_{i}(k+1)=v_{i}(k)+\Delta t\left(\sum_{j} q_{\text {in }}^{i, j}(k)-\sum_{l} q_{\text {out }}^{i, l}(k)\right),
$$

where $\Delta t$ is the sampling time and $k$ denotes the discretetime instant. The physical constraint related to the range of admissible water in the $i$-th storage element is expressed as

$$
\underline{v}_{i} \leq v_{i}(k) \leq \bar{v}_{i}, \quad \text { for all } k,
$$

where $\underline{v}_{i}$ and $\bar{v}_{i}$ denote minimum and maximum storage capacity.

\subsection{Actuators}

The manipulated flows through actuators represent manipulated variables, denoted as $q_{u}$. Both have lower and upper limits, which are taken as system constraints. As in formula (2), they are expressed as

$$
\underline{q u}_{i} \leq q_{u i}(k) \leq \bar{q}_{u}, \quad \text { for all } k,
$$

where $q_{u_{i}}$ and $\bar{q}_{u_{i}}$ denote the minimum and the maximum flow capacity, respectively. 


\subsection{Nodes}

These elements correspond to the network points where water flows are merged or split. Thus, the nodes represent mass balance relations, being modelled as equality constraints related to inflows and outflows. Expression of the mass conservation in these elements can be written as

$$
\sum_{j} q_{\text {in }}^{i, j}(k)=\sum_{h} q_{\text {out }}^{i, h}(k) .
$$

\subsection{River Reaches}

A single canal reach can be approximated by the approach proposed by [5] that leads to following relation between the upstream $\left(q_{u p s}\right)$ and downstream $\left(q_{d n s}\right)$ flows:

$$
q_{d n s}(k+1)=a_{1} q_{d n s}(k)+b_{0} q_{u p s}(k-d)
$$

where $d=\tau_{d} / T_{s}, \tau_{d}$ is the downstream transport delay, $T_{s}$ is the sampling time, $b_{0}=1-a_{1}$ and $a_{1}=e^{-\frac{T_{s}}{T}}$.

\subsection{Demand and Irrigation Sectors}

Demand and irrigation sectors represent water consume. It is considered as a measured disturbance of the system at a given time instant. The demand can be anticipated by a forecasting algorithm in [6] which is integrated within and run in parallel with MPC closed-loop architecture.

\section{MULTI-LAYER MPC SCHEME}

\subsection{Model Predictive Control}

The standard MPC problem based on the linear discretetime prediction model is considered as described in [7]:

$$
\begin{aligned}
x(k+1) & =A x(k)+B u(k) \\
y(k) & =C x(k)
\end{aligned}
$$

where $x(k) \in \mathbb{R}^{n_{x}}$ is state vector and $u(k) \in \mathbb{R}^{n_{u}}$ is vector of command variables, while $y(k) \in \mathbb{R}^{n_{y}}$ is vector of the measured output. Following the formalism provided by [7], the cost function is assumed to be quadratic and the constraints are in the form of linear inequalities, which constitute the following basic optimization problem (BOP):

$$
\begin{array}{ll} 
& \min _{\left(u(0 \mid k), \cdots, u\left(H_{p-1} \mid k\right)\right)} J(k) \\
\text { s.t. } & x(i+1 \mid k)=A x(i \mid k)+B u(i \mid k), \quad i=1, \cdots, H_{p} \\
& x(0 \mid k)=x_{k} \\
& x_{\min } \leq x(i \mid k) \leq x_{\max }, \quad i=1, \cdots, H_{p} \\
& u_{\min } \leq u(i \mid k) \leq u_{\max }, \quad i=0, \cdots, H_{p-1}
\end{array}
$$

As described $J$ is a performance index, representing operational goals of the system, $H_{p}$ is the prediction horizon, $x(0)$ is the initial condition of the state vector, $x_{\min }$ and $x_{\max }$ are minimal and maximal capacities of tanks, while $u_{\min }$ and $u_{\max }$ are known vectors defining the saturation constraints on inputs variables. Problem formula (7) can be recast as a Quadratic Programming (QP) problem, whose solution:

$$
\mathcal{U}^{*}(k) \triangleq\left[u^{*}(0 \mid k) \cdots u^{*}\left(H_{p}-1 \mid k\right)\right]^{T} \in \mathbb{R}^{H_{p} m \times 1}
$$

is a sequence of optimal control inputs that generates an admissible state sequence. At each sampling time $k$, Problem formula (7) is solved for the given measured/estimated current state $x(k)$. Only the first optimal move $u^{*}(0 \mid k)$ of the optimal sequence $\mathcal{U}^{*}(k)$ is applied to the process:

$$
u_{M P C}(k)=u^{*}(0 \mid k)
$$

the remaining optimal moves are discarded and the optimization is repeated at time $k+1$.

\subsection{Multi-layer Model Predictive Control}

This paper proposes a temporal multi-layer MPC scheme for the control of regional water networks [8].

The general principle of temporal multi-layer MPC is that decision of a higher layer has a wider temporal extent than the one of a lower layer. In this paper, a two-level structure related to the supply and transportation layers of a water network is proposed as shown in Figure 1.

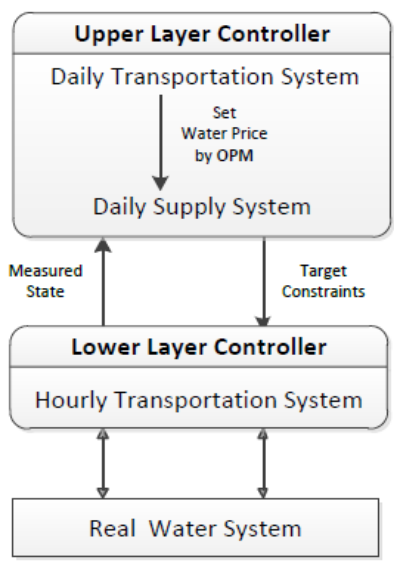

Figure 1: Temporal hierarchy Multi-layer MPC

The controllers of these two layers are operated in different goals and time scales. Inside the upper layer, OPM is used to reckon source price of the transportation layer.

\subsection{Optimal Path Method}

When optimize the supply system, transportation system would be simplified into a virtual demand with unitary price related with both treatment and electricity cost. In order to determine this unitary price, OPM is in needed[11]. The main steps and detail algorithm of OPM are provided:

step 1 Searching Exhaustive Paths: Find all possible paths from sources to demands detecting closed cycles to avoid infinite loops.

step 2 Choosing Optimal Path: Find optimal path from the set of all paths obtained in step 1 .

step 3 Calculating the source price: Calculate the source price taking into account the results of total cost and the water consumption in the optimal path obtained in step 2 . 


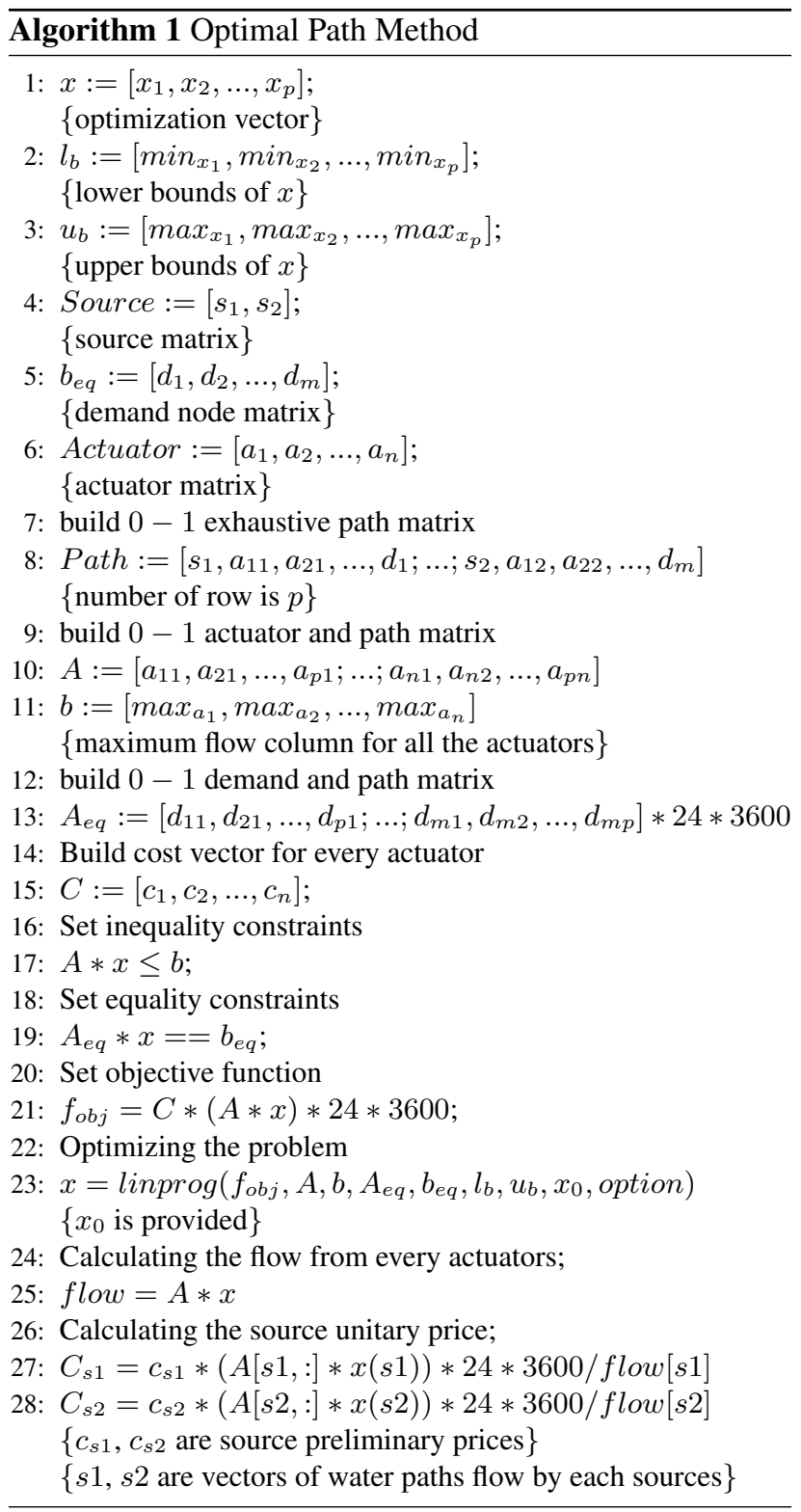

\subsubsection{Coordinating States}

As shown in Figure 1, the interaction between upper and lower layers relies on two elements:

- Measured state $\left(M_{s}\right)$ : which provides the daily demands to the supply layer by aggregating the hourly demands in the transportation layer.

- Target constraint $\left(T_{d}\right)$ : which expresses management policies at the supply layer to the transportation layer in the form of control constraints.

Measured State Measured state in every optimization iteration for the supply layer is obtained by aggregating the hourly demands in the transportation layer.

$$
M_{s}(k)=\sum_{m=1}^{24} d_{t}(k, m)
$$

where $d_{t}$ is hourly demand vector in transportation layer corresponding to the $k$-th day.

Target Constraints The goal of target constraints is transferring management policies from the upper to the lower layer. The management policies are calculated as:

$$
\sum_{m=1}^{24} u(k, m) \leq T_{d}(k)
$$

where $u$ is the shared control vector between supply and transportation layers.

This constraint is introduced to enforce that the amount of water decided to be transferred from the supply to the transportation layer by the upper layer MPC is respected by the lower layer MPC. Without such a constraint, the lower layer MPC would decide the amount of water ignoring the upper layer MPC policy.

The coordination structure is shown at Figure 2:

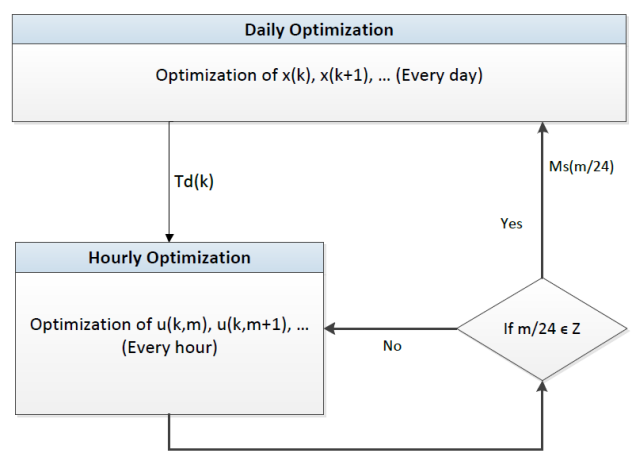

Figure 2: Upper and Lower layer optimizations of multi-layer MPC

\section{FORMULATION OF THE TEMPORAL MULTI-LAYER MPC SCHEME}

\subsection{State Space Model for Supply Layer}

The state space model of supply layer has two kinds of states and managed variables. First kind of state variable represents reservoirs and the managed variable corresponds to actuator flows:

$x(k+1)=A x(k)+B u(k)+B_{p}[d(k)-\varepsilon(k)], \quad k \in \mathbb{Z}$

where $x(k)$ is state variable represents volume, $u(k)$ is control which corresponds to actuator flows, $d(k)$ is disturbance corresponds to demands and $\varepsilon(k)$ is the slack variable for violated demand.

In model formula (12), $\varepsilon(k)$ is introduced to control the amount of demand which has not been satisfied.

The second kind of states and managed variable represent river flows with delays. For simplicity and brevity 
of the explanation, consider river reach model formula (5) as a transport delay [9]:

$$
q_{\text {out }_{i}}=q_{\text {in }_{i}}\left(k-\tau_{d}\right)
$$

where $\tau_{d}$ represents delay values. For time delays associated with flows within the network, the following auxiliary state equations are introduced:

$$
\begin{gathered}
x_{j, 1}(k+1)=q_{j}(k) \\
x_{j, i+1}(k+1)=x_{j, i}(k), i=1, \cdots, \tau_{d}
\end{gathered}
$$

where $x_{j, i}(k)$ are state variable representing flows, $q_{j}(k)$ mean flows, which is considered as part of managed variables and $\tau_{d}$ is the number of delays.

After integrating formula (14) and formula (15) with formula (12), we have

$$
\widetilde{x}(k+1)=\widetilde{A} \widetilde{x}(k)+\widetilde{B} \widetilde{u}(k)+\widetilde{B}_{p}[d(k)-\varepsilon(k)], \quad k \in \mathbb{Z}
$$

where

$$
\begin{aligned}
& \qquad \widetilde{x}(k)=\left[\begin{array}{c}
x(k) \\
x_{j}, i
\end{array}\right], \quad \widetilde{u}(k)=\left[\begin{array}{c}
u(k) \\
q_{j}(k)
\end{array}\right] \\
& \text { and } \widetilde{x}(k) \in \mathbb{R}^{\widetilde{n}_{x}}, \widetilde{u}(k) \in \mathbb{R}^{\widetilde{n}_{u}} \text {. }
\end{aligned}
$$

According to formula (2) and formula (3), all the variables are subject to the following inequality constraints:

$$
\begin{aligned}
& \widetilde{x}_{\min } \leq \widetilde{x}(k) \leq \widetilde{x}_{\max } \\
& \widetilde{u}_{\min } \leq \widetilde{u}(k) \leq \widetilde{u}_{\max } \\
& \varepsilon_{\min } \leq \varepsilon(k) \leq \varepsilon_{\max }
\end{aligned}
$$

where $\widetilde{x}_{\min }$ and $\widetilde{x}_{\max }$ are physical limitations of the reservoirs, while $\widetilde{u}_{\min }$ and $\widetilde{u}_{\max }$ are physical limitations of the river flows. The range of $\varepsilon_{\min }$ lies between zero and the related demand.

As described at Section II, balance at every node should be satisfied, where $E, E_{d}, E_{\widetilde{x}}$ are matrices obtained from topology of the water network:

$$
E \widetilde{u}+E_{d} d-E_{d} \varepsilon+E_{\widetilde{x}} \widetilde{x}=0
$$

The state space model of the transportation layer is the standard model in formula (6) where the states corresponds to tank volumes and the manipulated variables are the flows in pumps and valves. More details can be found in [1].

\subsection{Operational Goals}

\subsubsection{Operational Goals for Supply Layer}

The supply network is operated with a 30-day horizon, using daily time control interval. The main operational goals to be achieved in the supply network are:

goal 1 Operational safety $\left(J_{\text {safety }}\right)$ : Maintain appropriate water storage levels in dams for emergency-handling. goal 2 Demand management $\left(J_{\text {demand }}\right)$ : Fully satisfy urban demands while satisfy irrigation demands with slackness.

goal 3 Balance management ( $\left.J_{\text {balance }}\right)$ : Keep rivers or reservoirs consumed in a fair and balanced way in order to escape water deficits in the long run.

goal 4 Minimizing waste ( $\left.J_{\text {mwaste }}\right)$ : Minimize unnecessary water release from reservoirs which does not meet any demand and is eventually wasted.

goal 5 Environment conservation ( $\left.J_{\text {ecological }}\right)$ : Maintain water levels and ecological flows of rivers, which included in $J_{\text {safety }}$ during the calculation.

Above mentioned goals lead to the following function:

$$
\begin{aligned}
& J=J_{\text {safety }}+J_{\text {demand }}+J_{\text {mwaste }}+J_{\text {balance }} \\
& =\varepsilon_{\widetilde{x}}(k)^{\top} W_{\widetilde{x}} \varepsilon_{\widetilde{x}}(k)+\varepsilon(k)^{\top} W_{f} \varepsilon(k) \\
& +\left(\widetilde{u}_{i \ldots j}(k)-\widetilde{u}_{s}(k)\right)^{\top} W_{\widetilde{w}}\left(\widetilde{u}_{i \ldots j}(k)-\widetilde{u}_{s}(k)\right)
\end{aligned}
$$

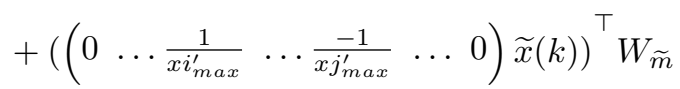

$$
\begin{aligned}
& \times\left(\left(\begin{array}{lllll}
0 & \ldots \frac{1}{x i_{\max }^{\prime}} & \ldots & \frac{-1}{x j_{\max }^{\prime}} \ldots & 0
\end{array}\right) \widetilde{x}(k)\right)
\end{aligned}
$$

where

$$
\begin{aligned}
\varepsilon_{\widetilde{x}}(k) & =\widetilde{x}(k)-\widetilde{x}_{r} \\
\widetilde{u} & =\Theta \Delta \widetilde{u}+\Pi \widetilde{u}(k-1) \\
\Delta \widetilde{u}(k) & =\widetilde{u}(k)-\widetilde{u}(k-1)
\end{aligned}
$$

$W_{\widetilde{x}}, W_{f}, W_{\widetilde{w}}, W_{\widetilde{x}}, W_{\widetilde{m}}$ are the related weights according to the priority policies established by network managers.

\subsubsection{Operational Goals for Transportation Layer}

The transportation network is operated with a 24-hour horizon, at hourly time interval. Besides goal 2 presented in above section, more goals need to be fulfilled:

goal 6 Cost reduction $\left(J_{\text {cost }}\right)$ : Minimize water cost during water supplying process.

goal 7 Control actions smoothness ( $\left.J_{\text {smoothness }}\right)$ : Smooth flow set-point variations for sustainable process operation.

Above mentioned goals lead to the following function:

$$
\begin{aligned}
J & =J_{\text {safety }}+J_{\text {smothness }}+J_{\text {cost }} \\
& =\varepsilon_{\widetilde{x}}(k)^{\top} W_{\widetilde{x}} \varepsilon_{\widetilde{x}}(k)+\Delta \widetilde{u}(k)^{\top} W_{\widetilde{u}} \Delta \widetilde{u}(k) \\
& +W_{a}\left(a_{1}+a_{2}(k)\right) \widetilde{u}(k)
\end{aligned}
$$

where

$$
\begin{aligned}
\varepsilon_{\widetilde{x}}(k) & =\widetilde{x}(k)-\widetilde{x}_{r} \\
\widetilde{u} & =\Theta \Delta \widetilde{u}+\Pi \widetilde{u}(k-1) \\
\Delta \widetilde{u}(k) & =\widetilde{u}(k)-\widetilde{u}(k-1)
\end{aligned}
$$

The vectors $a_{1}$ and $a_{2}$ contain the cost of water treatment and pumping, respectively. 


\subsection{Formulation of the optimization problem}

The objective formula (20) and objective formula (21) of the MPC problem can be formulated in the following way:

$$
J=z^{T} \Phi z+\phi^{T} z+c
$$

where

$$
z=\left[\begin{array}{lll}
\Delta \widetilde{u} & \varepsilon \widetilde{x} & \varepsilon
\end{array}\right]^{T}
$$

and $c$ is a constant value.

This allows to determine optimal control actions at each instant $k$ by solving a quadratic optimization problem (QP) in form as:

$$
\begin{gathered}
\min _{z} z^{\top} \Phi x+\phi^{\top} z \\
A_{1} z \leq b_{1} \\
A_{2} z=b_{2}
\end{gathered}
$$

\subsection{Formulation of Temporal Coordination Problem}

As explained in Section III, the goal for the temporal coordination algorithm is transferring management policies from the upper to the lower layer. Constraint formula (11) is added to the the lower layer MPC.

Constraints generated using the following algorithm:

step 1 after application of $n$ hourly control actions $u_{s}(m)$ corresponding to the $k$-th day, the total remaining water for this day will be: $T_{d}(k)-$

$$
\sum_{m=1}^{n} u(m)
$$

step 2 when limiting the control actions in the prediction horizon $L$, there is a part of control actions $u(m)$ that corresponds to hours of the current day $k$ that should be limited by $T_{d}(k)$, while the control actions correspond to hours of the next day $k+1$ that should be limited by $T_{d}(k)-\sum_{m=1}^{n} u(m)$.

step 3 the generated constraints are added as additional constraints of the BOP problem associated to the lower layer MPC.

\section{CASE STUDY: CATALUNYA REGIONAL WATER NETWORK}

In Figure 3, an aggregate model of Catalunya Regional Water Network is provided. According to the definition of functional decomposition, the supply layer, which composes rivers Llobregat, Ter and all the connected elements, lies on the two sides of Figure 3. The transportation layer, composed by metropolitan areas and also treatment, desalination plants inside them, is in the center of Figure 3.

\section{RESULTS}

\subsection{Supply Layer}

Table 1 provides detailed results and also comparison of water usages of the two rivers between MPC and the proposed multi-layer MPC scheme. In the table, $S$ means

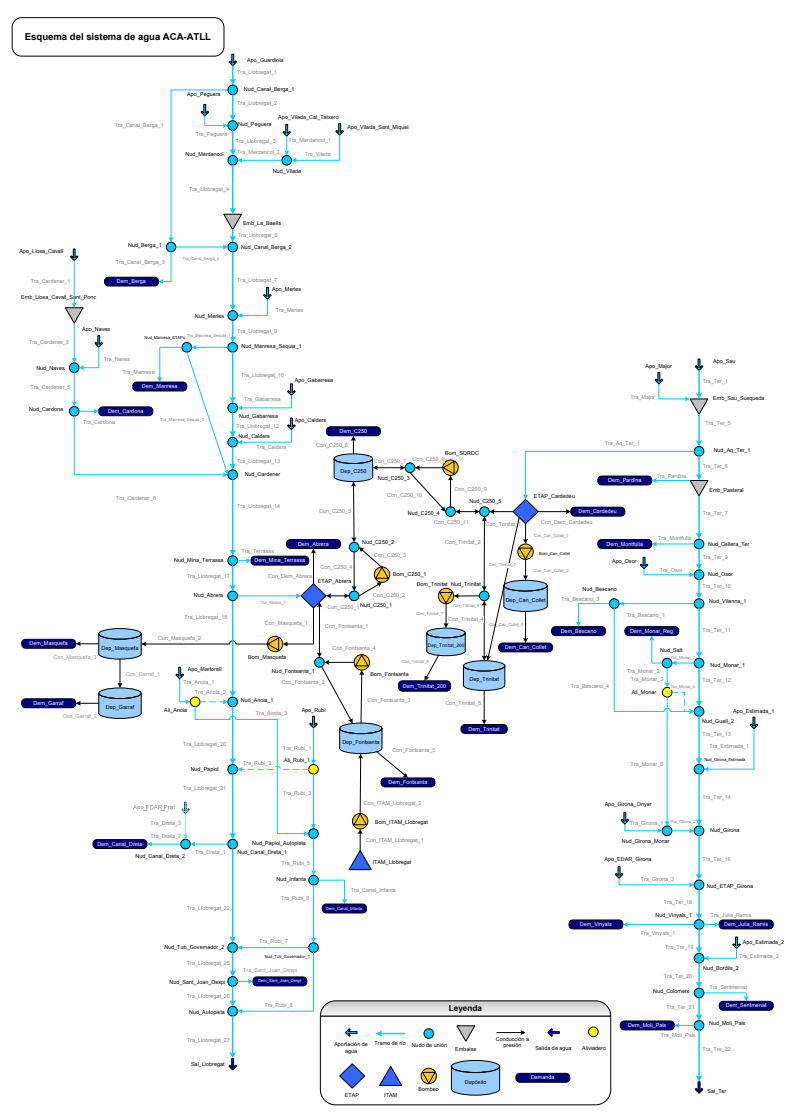

Figure 3: Aggregate diagram of Catalunya Regional Water Network.

outside sources flow into rivers, $F D$ means fixed demands which can not choose water source while $V D$ is the demand which can receive water from more than one river. $B D$ is water volume that has been consumed from each of the reservoirs and $P B$ is proportion of $B D . P R$ is the proportion of storage capacities of the two reservoirs. The similar values for $P B$ and $P R$ is what the multi-layer MPC wants to reach. And $S A$ is water supply ability in days of the whole water network before meeting deficit problems at the hypothesis of no rain and no water flow in from outside. The comparison proves that, multi-layer MPC scheme make the proportion of water usage from two rivers $(59.66 \%)$ much more proportional to their storage capacities $(53.48 \%)$. And what is more, the Catalunya Regional Water Network can supply water 62 days longer, which is a benefit for the sustainable usage of water resource in the long term perspective.

Table 1: Balancing comparison

\begin{tabular}{llllllll}
\hline Sc. & \multicolumn{7}{c}{ Multi-layer MPC } \\
\hline Es. & S & FD & VD & BD & PR & PB & SA \\
\hline L. & 3008 & 2981 & 724 & 710 & $59.66 \%$ & $53.48 \%$ & 2239 Days \\
T. & 3532 & 3518 & 1196 & 1169 & & \\
\hline Sc. & \multicolumn{7}{c}{ MPC } \\
\hline Es. & S & FD & VD & BD & PR & PB & SA \\
\hline L. & 3008 & 2981 & 7.6 & -19.4 & $-1.02 \%$ & $53.48 \%$ & \multirow{2}{*}{177 Days } \\
T. & 3532 & 3518 & 1914 & 1900 & & & \\
\hline
\end{tabular}




\subsection{Transportation Layer}

Figure 4 shows in the same plot the pump flow compared to its electricity fee. It can be noticed that pump sends more water to the reservoir at the lower price period and less or no water at the higher price period, which is consistency with the economical objective.

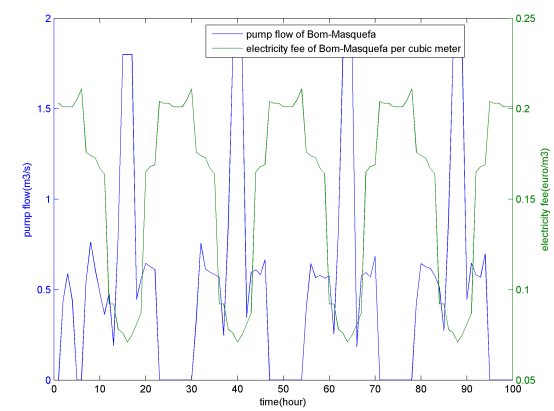

Figure 4: Pump flow with electricity price

\subsection{Coordination}

Figure 5 shows the amount of water consumed by the transportation layer from different rivers for satisfying the same demands before and after coordination, respectively. The two figures prove that average levels of water consumptions from two rivers are much closer after balance management.
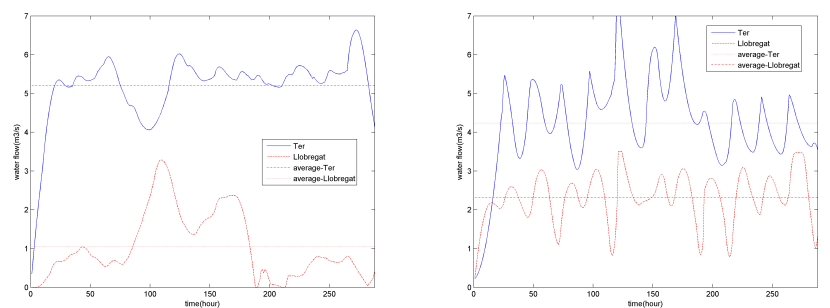

Figure 5: Flows of two rivers before and after temporal

Table 2 provides comparing results in economical cost over four days using three different control techniques: current control, which is the control using heuristic strategies by human operators; multi-layer MPC with OPM and multilayer MPC without OPM. It shows that, multi-layer MPC techniques are better than the current control. Besides that, multi-layer MPC with OPM is also better than that without OPM in electricity and also total cost but a little worse in water cost. That is because, without OPM, only water cost is considered, after introducing electricity item by OPM, the choice of source is not only decided by water cost, but also the transportation price. The introduction of OPM makes the whole water network have accurate and economical improvement.

\section{CONCLUSIONS}

In this paper, a multi-layer MPC scheme with multi-level coordination for regional water supply systems is proposed.
Table 2: Closed-loop performance results (all values in e.u.)

\begin{tabular}{ccccccccc}
\hline & Curr. & \multicolumn{3}{c}{ With OPM } & \multicolumn{3}{c}{ Without OPM } \\
Wat. & Ele. & Tot. & Wat. & Ele. & Tot. & Wat. & Ele. & Tot. \\
\hline 240 & 100 & 340 & 213 & 44 & 257 & 205 & 70 & 275 \\
239 & 106 & 345 & 237 & 47 & 284 & 210 & 61 & 271 \\
246 & 94 & 340 & 238 & 48 & 286 & 221 & 70 & 291 \\
264 & 110 & 374 & 253 & 66 & 319 & 245 & 94 & 339 \\
\hline \multicolumn{1}{c}{} & & $-5 \%$ & $-50 \%$ & $-18 \%$ & $-10 \%$ & $-28 \%$ & $-15 \%$ \\
\hline
\end{tabular}

OPM is introduced for accurate and economical improvement to the whole complex water network. The need of coordination derives from the fact that different networks in the water supply and transportation systems are operated according to different management goals, with different time horizon. While the management of the supply network is mainly concerned with long term safe-yield and ecological issues, the transportation layer must achieve economic goals in the short term, while meeting demands and operational constraints. The use of the modelling and coordination techniques proposed in this paper makes it possible to obtain short-term strategies which can effectively consider long-term objectives as well.

\section{REFERENCES}

[1] C. Ocampo-Martinez, V. Puig, G. Cembrano and J. Quevedo, Application of Predictive Control Strategies to the Management of Complex Networks in the Urban Water Cycle, IEEE Control Systems Magazine, 33(1): 15-45, 2013.

[2] M. Brdys and B. Ulanicki, Operational Control of Water Systems: Structures, algorithms and applications. UK: Prentice Hall International, 1994.

[3] P. J. Van Overloop, Model Predictive Control on Open Water Systems. Delft, The Netherlands: Delft University Press, 2006.

[4] G. Cembrano, J. Quevedo, V. Puig, R. Perez, J. Figueras, G. Ramon, P. Rodryguez, G. Barnet, M. Casas, J. M. Verdejo, A. Gil, J. Marti and H. Konig, Predictive optimal control of water supply and distribution, Control Engineering Practice, 2005.

[5] X. Litrico and V. Fromion, Simplified modeling of irrigation canals for controller design, Journal of Irrigation and Drainage Engineering, 130(5): 373-383, 2004.

[6] J. Quevedo, V. Puig, G. Cembrano and J. Blanch, Validation and reconstruction of flow meter data in the Barcelona water distribution network, Control Engineering Practice, 11(6): 640-651, 2010.06.

[7] J. M. Maciejowski, Predictive Control with Constraints. Great Britain: Prentice Hall, 2002.

[8] M. A. Brdys and P. Tatjewski, Iterative Algorithms for Multilayer Optimizing Control. London: Imperial College Press, 2005.

[9] R. Evans, L. Li, I. Mareels, N. Okello, M. Pham, W. Qiu and S. K. Saleem, Real-time Optimal Control of River Basin Networks, in the 18th IFAC World Congress, "11459-11464", 2011.

[10] C. C. Sun, V. Puig and G. Cembrano, Multi-layer Model Predictive Control of Regional Water Networks: Application to the Catalunya Case Study, in 52nd Conference on Decision and Control, Florence, to appear, 2013.

[11] W. Cheng, N. Hsu, W. Cheng and William W.-G. Yeh, A flow path model for regional water distribution optimization, Water resources research, 45: W09411, 2009. 